\title{
Rutherford Backscattering Angular Yields under Channeling Conditions for $\mathbf{H}^{+}$and $\mathbf{H}^{0}$ Projectiles ${ }^{*}$
}

\author{
J. Czerbniak ${ }^{\dagger}$, M. Moneta \\ Uniwersytet Łódzki, Katedra Fizyki Ciała Stałego, Zakład Fizyki Powierzchni \\ Pomorska 149, 90-236 Łódź, Poland
}

AND G. PUdŁOWSKI

Wyższa Szkoła Informatyki, Rzgowska 17A, 93-008 Łódź, Poland

(Received September 25, 2001; revised version December 4, 2001;

in final form April 5, 2002)

\begin{abstract}
The Rutherford backscattering angular scattering yields for $100 \mathrm{keV}$ $\mathrm{H}^{+}$and $\mathrm{H}^{0}$ projectiles from Si crystal was measured. The analysis of results using Monte Carlo simulations leads to the conclusion that $\mathrm{H}^{0}$ beam suffers smaller dispersion than $\mathrm{H}^{+}$beam, when crossing crystal surface. It means that the $\mathrm{H}^{0}$ scattering probability is smaller at large impact parameters. For greater penetration depth the channeling process turns out to be the same.
\end{abstract}

PACS numbers: 71.45.Gm

\section{Introduction}

For swift ions the electronic stopping cross-section $S_{e}$ is described by the Bethe theory and is proportional to the square of ion atomic number $S_{\mathrm{e}} \propto Z_{1}^{2}$. For medium velocity ions it is necessary to introduce the concept of effective charge $Z^{*}$ and in this case $S_{\mathrm{e}} \propto Z^{* 2}[1]$. In general, the effective charge of ions in matter is different from the ion atomic number. For ions of energy $1 \mathrm{MeV} / \mathrm{a} . \mathrm{m} . \mathrm{u}$. the charge distribution reaches equilibrium distribution on the distance of $L \propto 10 \mathrm{~nm}[2,3]$. With

*supported by Uniwersytet Łódzki, grant 505/445 (2001)

$\dagger$ corresponding author; e-mail: czermich@krysia.uni.lodz.pl 
decrease in ion velocity $L$ also decreases, and for the energy 0.4-0.6 MeV/a.m.u. it reaches $L \propto 1 \mathrm{~nm}[4,5]$. The energy losses along the main crystallographic directions, in channeling conditions, are much smaller than the energy losses in disordered structures due to the lowering of the probability excitation and charge exchange [6]. The characteristic distance for reaching the equilibrium charge distribution for $0.38 \mathrm{MeV} \mathrm{He}{ }^{+}$and $\mathrm{He}^{2+}$ ions is about $2 \mathrm{~nm}$, i.e. several lattice constants [7]. It is difficult to estimate the contribution to the energy loss from the first atomic surface layer and relate it to the contribution that comes from the ion motion in the bulk lattice. A possible experimental method to find the relation is a detailed analysis of the Rutherford backscattering (RBS) energy spectra in SIMOX-like (separation by implantation of oxygen) structures [8]. Also, the sensitivity of the angular RBS spectra on the angle of ions incidence with respect to crystallographic axis and planes is commonly known. Particularly, such spectra depend on parameters of the ion beam incidence in the crystal. The initial ion beam divergence and the surface structure strongly modify the measured spectra. It is expected that the analysis of angular RBS spectra measured as functions of the initial charge state of the incident ion beam enables getting information on the process of equilibration of the charge state distribution in the subsurface regions for medium energy ions. We used the RBS technique in order to get backscattering angular spectra for $100 \mathrm{keV} \mathrm{H}^{+}$and $\mathrm{H}^{0}$ projectiles channeled in single Si crystal.

\section{Experimental procedure}

The experimental arrangement and the sample preparation used in this experiment were described elsewhere [9]. The incident ion beam consisting of protons $\mathrm{H}^{+}$and hydrogen atoms $\mathrm{H}^{0}$ of the angular divergence of $0.02 \mathrm{deg}$ was directed onto $\mathrm{Si}(001)$ single crystal mounted on a three-axis goniometer of $0.01 \mathrm{deg}$ setting accuracy. The beam spot of $0.5 \mathrm{~mm}$ diameter was shifted by $0.3 \mathrm{~mm}$ with respect to the rotation axis of the crystal in order to minimise radiation damage. The backscattered ions were registered with a semiconductor detector placed at an angle of $130 \mathrm{deg}$. The detector solid angle was 0.01 srad and the energy resolution FWHM $=10 \mathrm{keV}$. By selecting appropriate energy threshold for detected signals it was possible to detect backscattered ions from the depth interval of up to $40 \mathrm{~nm}$. This depth corresponds to the channeling oscillation length of ions along main crystallographic planes.

In order to increase ion beam neutralization a neutralizer was mounted in the reaction chamber just before the final collimating slot. The neutralizer was built from $70 \mathrm{~cm}$ long tube with controlled gas pressure ranging from $10^{-2}$ to $10^{-3}$ Torr. A switching on of the neutralizer produced $15-20 \%$ neutralization of the ion beam. In order to apply neutral projectiles only the charged fraction was electrostatically removed from the beam.

The surface of Si single crystal was cleaned by oxidation at the temperature of $1000 \mathrm{~K}$ in the presence of $\mathrm{H}_{2} \mathrm{O}$ vapour for 5 min and subsequent removal of 
the resultant $\mathrm{SiO}_{2}$ layer in $15 \% \mathrm{HF}$ acid. After that the crystal was immediately mounted on the goniometer in the reaction chamber.

The angular yields of random backscattered projectiles were measured for two full rotations of the crystal (12000 points). The tilt angle of the rotation axis $\langle 001\rangle$ with respect to ion beam was $\theta=42 \mathrm{deg}$. The neutralization was switched on producing $\mathrm{H}^{0}$ during one crystal rotation and then during the next one rotation it was off producing $\mathrm{H}^{+}$. Due to the crystal symmetry in (001) orientation after every 90 deg of rotation the same RBS angular scan was registered. Thus, the comparison of subsequent scans enabled estimation of the time stability of measurements. In the case of $\mathrm{H}^{+}$no radiation damage was found in the angular scan during one crystal rotation $(3200 \mathrm{~s})$, for the beam current of the order of $1 \mathrm{nA}$ on the target.

Since for low energy incident particles $(100 \mathrm{keV})$ the widths of channeling dips are large, the determination of so-called "random" level was impossible. As a consequence each part of the angular scan contains minima corresponding to the channeling in the high index axial or planar directions. For these directions sensitivity of the channeling effect to the angular divergence of the beam after transmission through the crystal surface increases. Thus the continuum potential model [10] does not allow for sufficiently precise theoretical calculation of large parts of angular scans. The influence of the crystal surface on the RBS angular spectra were analysed by comparing the measured spectra with the spectra obtained by computer simulation of the channeling.

\section{Simulation}

In the Monte Carlo simulation of the channeling process the channeling cell was selected in $\langle 001\rangle$ direction as described in Ref. [11]. The projectile trajectory was determined on the basis of binary collision model with the step of calculation $l=0.25 a / \cos \theta$, where $a$ is the lattice constant for Si, $\theta$ is the tilt angle of the rotation axis with respect to the ion beam direction. The scattering angle was calculated from the magic formula [12] using the Ziegler-Biersack-Littmark (ZBL) potential. In each step of calculation the temporary position of the nearest-neighbour thermally vibrating atom was determined from the normal distribution. For $293 \mathrm{~K}$ the one-dimensional thermal vibration amplitude of Si atom is $0.00756 \mathrm{~nm}$. The breaking point correction for rectilinear parts of trajectory was taken into account by calculating that the incident and outgoing asymptotes of trajectory cross at a point which is in the distance $s$ before the projection of the scattering centre on incident trajectory

$$
s=\frac{2 m_{1}}{m_{1}+m_{2}} \tau+\frac{m_{2}-m_{1}}{m_{1}+m_{2}} b \tan (\alpha / 2),
$$

where $\tau$ is the time integral, $b$ is the scattering parameter, $\alpha$ is the scattering angle. For $m_{1} \ll m_{2}, s=b \tan (\alpha / 2)$. The energy losses due to interaction with electrons were determined for each scattering using the Dettman-Robinson model 
[13], i.e. by taking into account separately the shell and valence electron contributions and close and distant collisions. The change of trajectory of motion due to close collisions with electrons $\Delta \alpha_{\text {scatt }}=m_{\mathrm{e}} \Delta E_{\text {close }} / m_{1} E$ was taken into account by determining the scattering angle from normal distribution $N(\alpha, \Delta \alpha)$. The nuclear encounter probability (NEP) [14] was calculated for each scattering event and stored as a function of the collision number and separately as a function of the energy loss. In the latter the NEP was corrected according to the increase in the scattering cross-section with decrease in ion energy. The straight line trajectory of ion motion from the point of scattering to the crystal surface (and to the detector) along with constant energy loss per unit path length were assumed. The straggling on the outgoing path and the energy resolution of the detector were taken into account. The structure of crystal surface was taken into account by generating random positions of $\mathrm{Si}$ and $\mathrm{O}$ atoms for the first and subsequent layers of the selected channeling cell. On the basis of calculated $\operatorname{NEP}(E)$ the average values of NEP for the energy region corresponding to the energy resolution of spectrometric setup and for the subsequent values of the rotation angle $\omega$ for a given tilt angle $\theta$ were determined. In this manner the code generated results correspond exactly to the geometry and experimental conditions. Next, the calculated angular distributions were related to the experimental scans. The normalizing factor was the ratio of counts from the analyzed region of crystal rotation angle $\omega$ for calculated and measured spectra, to NEP for the same region of rotation. A sequence of spectra was generated for randomly distributed $\mathrm{Si}$ and $\mathrm{O}$ atoms (creating $\mathrm{SiO}_{2}$ ) in subsequent layers and the theoretical and experimental spectra were fitted by means of $\chi^{2}$ criterion.

\section{Results and data analysis}

A typical fragment of angular RBS angular yields for $\mathrm{H}^{+}$and $\mathrm{H}^{0}$ particle beam incident on $\mathrm{Si}(001)$ with $\theta=42 \mathrm{deg}$ is shown in Fig. 1. Both yields were measured for the same sample. A more detailed structure of the $\mathrm{H}^{0}$ angular scan than that of the $\mathrm{H}^{+}$scan was found in regions of minima and in shoulder regions. These differences were recorded for all samples, but they disappear for penetration depth of 100-150 nm. It means that the dechanneling effect prevails over surface effects causing ion beam dispersion. The analysis of minima for $\mathbf{H}^{0}$ and $\mathbf{H}^{+}$gives, within the statistical error, the same angular width. Also, $\chi_{\min }$ determined with respect to arbitrary accepted "random" level gives, within the statistical error, the same values. The angular widths of dips in the "shoulder" regions are also the same. It means that for low index crystallographic planes the averaged height of the potential barrier in the model of continuous potential [10] is the same. Next, it means that the channeling of $\mathrm{H}^{+}$in the crystal is independent of the initial charge state of the beam.

The Monte Carlo simulation code was used to find measurement parameters which give the best agreement with experimental results, as shown in Fig. 2. The 


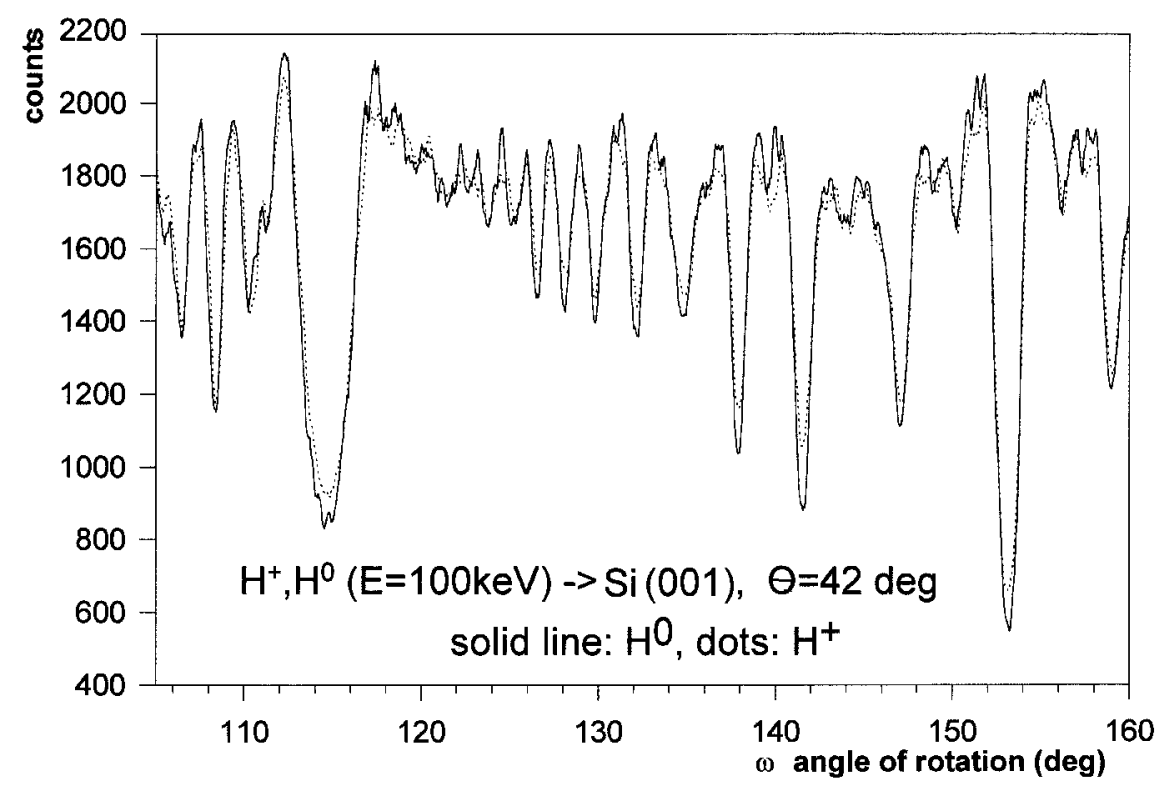

Fig. 1. Experimental RBS angular yields for $100 \mathrm{keV} \mathrm{H} \mathrm{H}^{+}-$dots and $\mathrm{H}^{0}-$ solid line. The tilt angle $\theta=42 \mathrm{deg}$ of the rotation axis to the ion beam line.

parameter to be determined was the number $n$ of centres in the first layers of the crystal giving contribution to the low angle scattering, i.e. leading to the increased divergence of the incident ion beam. For $\mathrm{H}^{0}$ the best agreement was obtained for density of scattering centres $n_{0}=2 \times 10^{14}$ at. $\mathrm{cm}^{-2}$ corresponding to Gaussian spreading of the ion beam of $0.1 \mathrm{deg}$, and for $\mathrm{H}^{+}$the density is $n_{1}=2 \times 10^{15}$ at. $\mathrm{cm}^{-2}$. The difference is interpreted that $\mathrm{H}^{0}$ interact with disordered surface layer as soon as it loses its electron. However, in experiment, it was accepted as a good approximation that in both cases (i.e. $\mathrm{H}^{+}$and $\mathrm{H}^{0}$ ) the disordered surface layer was the same. In the case of $\mathrm{H}^{+}$ions the simulation gives the best agreement with experiment, if first four surface layers (it corresponds to $0.4 \mathrm{~nm}$ looking along $\langle 001\rangle$ direction) are disordered. Even if we do not know exact parameters of this layer, we find that the scattering probability is larger in the case of $\mathrm{H}^{+}$than in that of $\mathrm{H}^{0}$. The ion beam reaches equilibrium charge distribution after three scatterings, and the following motion is independent of the initial charge state.

\section{Conclusions}

In the presented work the channeling of $100 \mathrm{keV} \mathrm{H^{0 }}$ and $\mathrm{H}^{+}$particles was analyzed. The classical RBS experimental setup was used to get backscattered angular scans from typical Si single crystal. Experimental results with the aid of Monte Carlo simulation and fitting procedure reveal that in case of $\mathrm{H}^{0}$ the path 


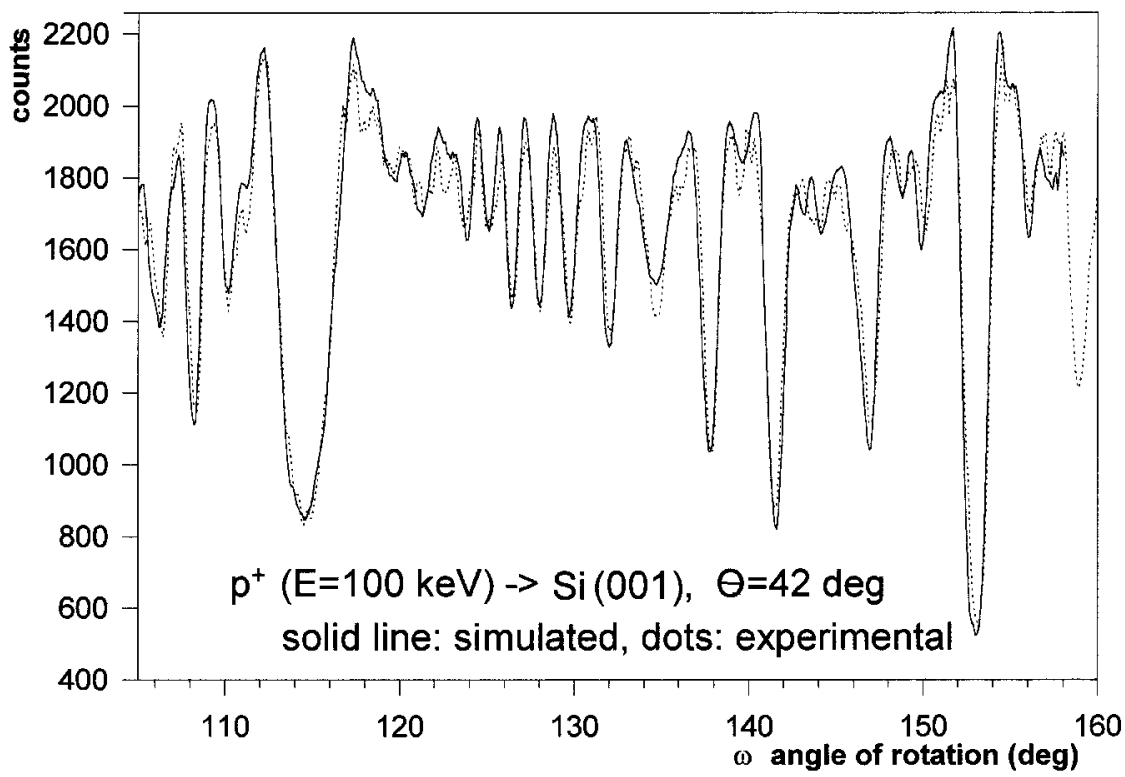

Fig. 2. Simulated (solid line) and experimental (dots) RBS angular yields for $\mathrm{H}^{+}$. For simulation $n=2 \times 10^{14}$ atoms $/ \mathrm{cm}^{2}$ of Si and $\mathrm{O}$ atoms randomly distributed in the first $\mathrm{Si}(001)$ layer was assumed.

for loss of electron is $0.4 \mathrm{~nm}$. The scattering probability before crossing the crystal surface and in the subsurface region is larger in the case of $\mathrm{H}^{+}$than in that of $\mathrm{H}^{0}$. Also, the detailed analysis of large fragments of angular spectra may be treated as an additional method for analysis of structure of subsurface regions.

\section{References}

[1] W. Brandt, M. Kitagawa, Phys. Rev. B 25, 5631 (1982).

[2] N.E.B. Cowern, P.M. Read, C.J. Sofield, L.B. Bridwell, M.W. Lucas, Phys. Rev. A 30, 1682 (1984).

[3] A.F. Burenkow, F.F. Komarov, S.A. Fedotov, Nucl. Instrum. Methods Phys. Res. $B$ 83, 1682 (1992).

[4] S. Jans, P. Oberschachtsiek, S. Kalbitzer, M. Behar, Nucl. Instr. Methods Phys. Res. B 83, 1 (1993).

[5] T.M. Buck, G.H. Weatley, L.C. Feldman, Surf. Sci. 35, 345 (1973).

[6] G. de M. Azevedo, J.R.A. Kaschny, M. Behar, P.L. Grande, Ch. Klatt, S. Kalbizer, Nucl. Instrum. Methods Phys. Res. B 161, 96 (2000).

[7] J.H.R. dos Santos, P.L. Grande, H. Boudinov, M. Behar, in: Proc. Conf. on Ion Implantation Technology-94, Eds. S. Coffa, G. Perla, F. Priolo, E. Rimini, Elsevier, Amsterdam 1995, p. 771. 
[8] G. de M. Azevedo, P.L. Grande, M. Behar, J.F. Dias, Phys. Rev. Lett. 86, 1483 (2001).

[9] J. Czerbniak, M. Moneta, B. Bawlowski, M. Piwowarski, Phys. Status Solidi B 174, 773 (1988).

[10] J. Lindhard, Kgl. Danske Vid. Selsk. Mat.-Fys. Medd 28, no. 8 (1954).

[11] P.J.M. Smulders, D.O. Boerma, Nucl. Instr. Methods Phys. Res. B 29, 471 (1987).

[12] J.F. Ziegler, J.P. Biersack, U. Littmark, The Stopping and Range of Ions in Matter, Pergamon Press, New York 1985.

[13] K. Dettman, M.T. Robinson, Phys. Rev. B 10, 1 (1974).

[14] J. Barret, Phys. Rev. B 20, 3535 (1979). 